\title{
Análise da Dispersão Cromática em Um Novo Modelo de Fibra Óptica Usando Algoritmos Genéticos
}

\author{
Diego S. Bezerra e José P. da Silva
}

\begin{abstract}
Resumo-Algoritmos genéticos em conjunto com o método dos elementos finitos são utilizados na otimização de um novo modelo de fibra óptica com dispersão cromática ultra-plana. A estrutura é formada pela inclusão de um pequeno furo de ar no centro do núcleo de uma fibra óptica convencional e exibe uma dispersão cromática de $\pm 0,9$ ps/(km.mm) para o modelo otimizado.
\end{abstract}

Palavras-Chave-Algoritmos Genéticos, dispersão cromática, elementos finitos.

Abstract-Genetic Algorithms in conjunction with the finite element method are used to improve a new optical fiber model with ultra-flattened dispersion. The structure is formed by introducing a small air-hole at the core of a conventional fiber and exhibit a $\pm 0.9 \mathrm{ps} /(\mathbf{k m} . \mathrm{mm})$ chromatic dispersion for the optimized model.

Keywords-Genetic Algoritms, chromatic dispersion, finite elements.

\section{INTRODUÇÃO}

A busca por novos modelos de fibras ópticas que apresentam baixa dispersão cromática para uma extensa faixa de freqüência tem atraído o interesse de um grande número de pesquisadores da área de fotônica nos últimos anos, sobretudo por representarem um excelente meio de transmissão para grandes links de comunicação [1]-[3]. Nesse contexto, vários dispositivos condutores de luz têm sido relatados na literatura com ênfase para as fibras de cristais fotônicos, as quais apresentam, em alguns modelos, uma dispersão cromática ultraplana [2]. Neste trabalho, o método dos elementos finitos em conjunto com a técnica de otimização baseada em algoritmos genéticos (AG) são usados na análise de um novo modelo de fibra óptica que apresenta dispersão cromática ultra-plana em vários comprimentos de onda.

A estrutura proposta caracteriza-se pela inclusão de um furo de ar de raio muito pequeno no centro do núcleo de uma fibra convencional, Fig. 2. Os diâmetros do furo de ar e do núcleo da fibra são otimizados através de um algoritmo genético programado para obter uma fibra óptica com dispersão cromática ultra-plana para uma extensa faixa de frequiências. Na próxima seção, a formulação por elementos finitos é resumidamente explicada, noções sobre algoritmos genéticos são descritas na seção 3, resultados numéricos são exibidos na seção 4, e finalmente são apresentadas as conclusões deste trabalho.

Diego S. Bezerra e José P. da Silva, Departamento de Ciências Ambientais e Tecnológicas - DCAT, Universidade Federal Rural do Semi-Árido, MossoróRN, Brasil, E-mails: diego.bezerra@mcc.ufersa.edu.br, patroc@ufersa.edu.br. Este trabalho foi financiado pela FAPERN e CNPq (563271/2008-6).

\section{Formulação Por Elementos Finitos}

O método dos elementos finitos é amplamente reconhecido como uma poderosa ferramenta numérica para análise de dispositivos ópticos [4]-[8]. A aproximação por elementos finitos vetoriais para análise das características de propagação em fibras ópticas com alta precisão é abordada em [8].

Em sistemas de comunicações ópticas, o conhecimento da dispersão cromática $D$ torna-se extremamente importante para determinar a qualidade da transmissão do pulso, devido este fator ser diretamente responsável pela largura do mesmo. Por outro lado, $D$ é proporcional à derivada segunda da constante de propagação ou índice de refração efetivo $n_{\text {eff }}$, desta forma, uma ferramenta numérica altamente precisa, como o método dos elementos finitos [9], torna-se necessário para obter o $n_{\text {eff }}$. Aqui, além da aplicação do método numérico, os coeficientes de Sellmeier, foram usados na formulação para analisar a dispersão em diferentes frequiências.

Os coeficientes de Sellmeier são aplicados diretamente na formulação vetorial por elementos finitos, que, em síntese, é obtida partindo-se da equação vetorial de Helmholtz em duas dimensões e considerando camadas perfeitamente casadas (PMLs) para evitar reflexões indejadas. Desta forma, pode-se escrever:

$$
\nabla \times(\overline{\bar{k}} \nabla \times \vec{H})-k_{0}^{2} \vec{H}=0
$$

onde $\overline{\bar{k}}=1 / \overline{\bar{\varepsilon}}$ e $\overline{\bar{\varepsilon}}$ representa o tensor permissividade relativa. Considerando o meio dielétrico com anisotropia transversal e definindo $\hat{u}_{x}, \hat{u}_{y}$ e $\hat{u}_{z}$ associados com as direções $x, y$ e $z$ respectivamente, o tensor permissividade $\overline{\bar{\varepsilon}}$ pode ser escrito como $\bar{\varepsilon}=\varepsilon_{x x} \hat{u}_{x} \hat{u}_{x}+\varepsilon_{x y} \hat{u}_{x} \hat{u}_{y}+\varepsilon_{y x} \hat{u}_{y} \hat{u}_{x}+\varepsilon_{z z} \hat{u}_{z} \hat{u}_{z}$. Usando o procedimento convencional por elementos finitos baseado no método de Galerkin aplicados na equação (1), após algumas manipulações algébricas e assumindo que tanto o meio como os campos variam muito lentamente ao longo da direção de propagação [9], o seguinte sistema matricial é obtido:

$$
[A]\{\phi\}=n_{\text {eff }}^{2}[B]\{\phi\}
$$

onde $[A]$ e $[B]$ são matrizes esparsas e complexas. A equação (2) é eficientemente resolvida através do método da interação de subespaços. Em adição, é importante ressaltar que o perfil de índice de refração $n(r, \lambda)$ de uma fibra óptica pode ser escrito como $n(r, \lambda)=\eta(r) n_{s}(\lambda)$, onde $n_{s}(\lambda)$ é o índice de refração da sílica pura e $\eta(r)$ é o índice de refração normalizado, o qual é função apenas da coordenada radial. 
Aqui, as constantes de Sellmeier para o índice de refração da sílica pura $n_{s}(\lambda)$ dadas em [10] são consideradas. Desta forma, a dispersão do material é incluída diretamente nos cálculos. Os coeficientes de Sellmeier foram usados porque eles podem ajustar-se a medidas experimentais dos índices de refração da sílica. O $n_{\text {eff }}$ é obtido a partir da equação (2), considerando uma faixa de comprimentos de onda, e a dispersão cromática pode ser diretamente calculada a partir da seguinte expressão: $D=-(\lambda / c)\left(d^{2} n_{\text {eff }} / d \lambda^{2}\right)$, onde $c$ representa a velocidade da luz no espaço livre.

\section{Algoritmos Genéticos}

Algoritmos genéticos são estruturas computacionais baseadas na teoria da evolução das espécies apresentada por Darwin. A teoria defende que a evolução ocorre devido à melhor adequação de determinados indivíduos ao ambiente ao qual pertencem. Estes indivíduos compõem uma população que procria ao longo do tempo, dando origem a novos indivíduos que apresentam carga genética dos pais, bem como alterações devido a possíveis mutações. Indivíduos externos podem juntar-se à população, mantendo assim, a diversidade genética. Então, a natureza impõe obstáculos à sobrevivência, causando a seleção dos espécimes mais adaptados, reiniciando todo o ciclo.

Algoritmos genéticos são utilizados na otimização de problemas complexos e apresentam uma grande similaridade em sua execução, contudo, apenas isto torna dois ou mais AG similares. Estas partes em comum caracterizam-se pela utilização de uma população, representada por indivíduos, que evolui no decorrer da aplicação, utilizando-se dos métodos de criação, mutação, crossing-over, avaliação e seleção [3]. Como cada algoritmo visa solucionar um determinado problema, o programador necessita dominar sua realidade para que seja possível uma boa representação da mesma em um ambiente computacional. Isto é bastante visível na representação de um caso, ou indivíduo, que possui uma estrutura (genes) única.

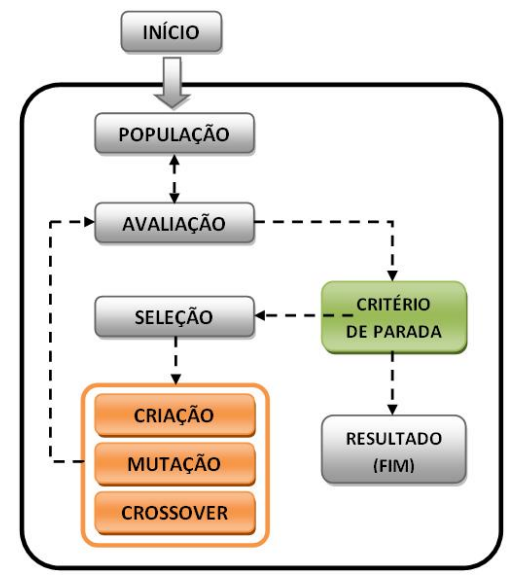

Fig. 1. Fluxograma do algoritmo genético.

Definida a estrutura do indivíduo, o algoritmo trata de gerar uma população inicial com a função de criação. Os valores aqui atribuídos aos genes dos indivíduos são aleatórios respeitando uma faixa de interesse. Em seguida esta população é avaliada por meio da função de avaliação, aplicando-se a seleção com o intuito de descartar os piores indivíduos. Concluidas as etapas anteriores, se inicia o laço onde novos indivíduos serão gerados (agora usando também as funções de mutação e crossing-over), avaliados e selecionados até que o critério de parada seja satisfeito. Este critério pode ser um determinado número de gerações ou pode ser um valor de referência considerado satisfatório. A Fig. 1 mostra o fluxograma para o código genético desenvolvido.

\section{RESUlTADOS}

Para obter a fibra com características de dispersão cromática ultra-plana, utilizou-se um código genético como o mostrado na Fig. 1. Os parâmetros otimizados da estrutura mostrada na Fig. 2, usados nesta análise, foram: $\eta_{1}=1,05190, \eta_{2}=1,0$, $r_{1}=0,194 \mu \mathrm{m}$ e $r_{2}=1,8 \mu \mathrm{m}$.
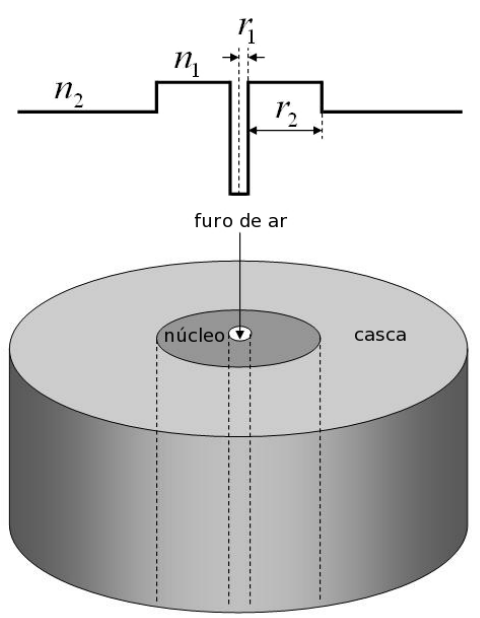

Fig. 2. Perfil de índice de refração da fibra óptica com furo de ar no centro do núcleo.

A simulação fez uso de uma malha discretizada por 5.500 elementos quadráticos dentro de uma janela computacional de $6 \mu \mathrm{m}$ (direção $x$ ) por $6 \mu \mathrm{m}$ (direção $y$ ). A dispersão cromática obtida é ultra-plana e varia entre $\pm 0,9 \mathrm{ps} /(\mathrm{km} . \mathrm{nm})$ para uma faixa de comprimentos de onda que vai de aproximadamente $1,25 \mu \mathrm{m}$ a $2,12 \mu \mathrm{m}$. É importante ressaltar que para o resultado não otimizado usado a título de comparação na Fig. 3, os parâmetros para a fibra foram aqueles apresentados em [4]. $\mathrm{Na}$ estrutura proposta neste trabalho, Fig. 2, os parâmetros $\eta_{1}, \eta_{2}, r_{1}$ e $r_{2}$ foram otimizados usando algoritmo genético enquanto que os parâmetros para a mesma estrutura usados em [4] foram obtidos empiricamente.

Resultados preliminares mostraram que o uso de malhas mais refinadas com número de elementos muito grande não contribui com a precisão dos resultados alcançados para a estrutura otimizada por algoritmo genético. A estrutura otimizada foi obtida em um tempo total de simulação de aproximadamente 3 horas em um computador Intel ${ }^{\circledR}$ Core $^{\mathrm{TM}}$ 2 Duo com 1,8 GHz. O algoritmo foi executado em um sistema operacional Linux com arquitetura x86_64 (64bits), que mostrou ser aproximadamente $8 \%$ mais rápida que a arquitetura x86 (32bits). Novos testes estão sendo feitos com o 


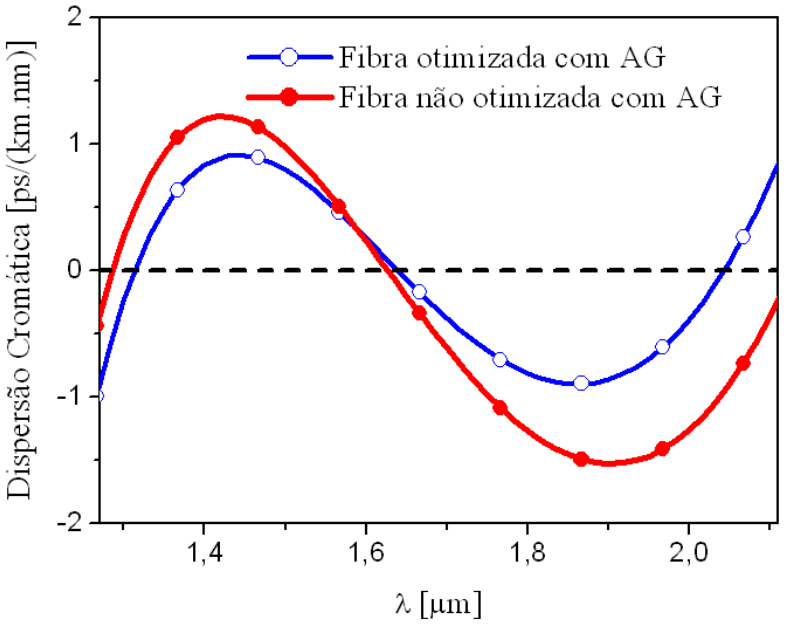

Fig. 3. Dispersão cromática para a estrutura mostrada na Fig. 2.

uso de processamento paralelo usando consoles PlayStation ${ }^{\circledR}$ 3 formando um Cluster no ambiente Linux com a intenção de reduzir o tempo de processamento.

A Fig. 4 mostra as curvas de dispersão cromática para a fibra óptica em análise, sem o furo de ar introduzido no centro do núcleo da fibra e com a introdução do pequeno furo de ar, considerando a estrutura otimizada. É importante salientar que pequenas alterações nas dimensões da estrutura poderão influenciar bastante nas características de dispersão da fibra, deste modo, a aplicação de algoritmos genéticos representa um avanço no que se refere à obtenção dos parâmetros da estrutura mostrada na Fig. 2. Além disso, o modelo da fibra aqui analisada é de fácil construção quando comparada a alguns modelos de Fibras de Cristais Fotônicos (PCFs) [6], utilizadas para obtenção de dispersões cromáticas ultra-planas.

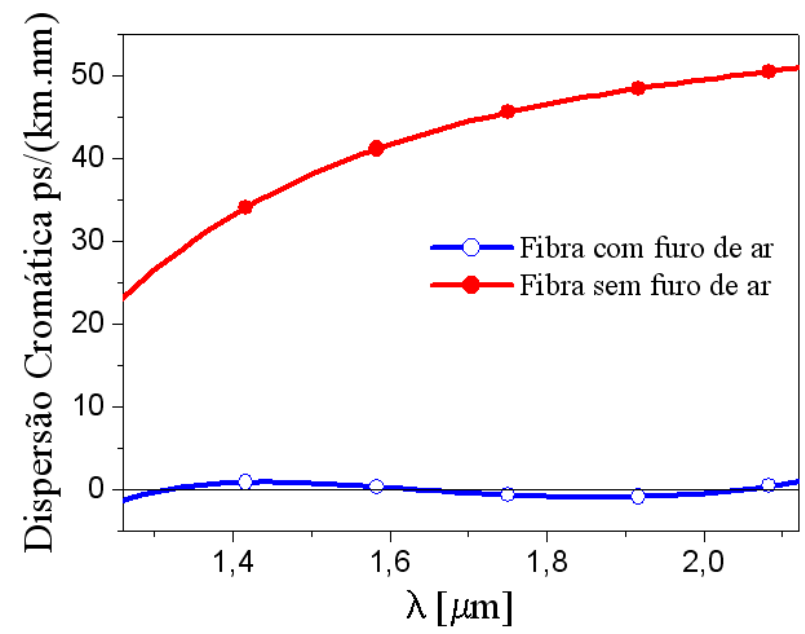

Fig. 4. Dispersão cromática para a estrutura mostrada na Fig. 2, sem o furo de ar e com a inclusão do furo de ar.

A Fig. 5 mostra a variação da área efetiva do modo fundamental em função do comprimento de onda. A área efetiva, $A_{\text {eff }}$, é uma medida quantitativa da área efetivamente coberta pelo modo da fibra em relação às dimensões transversais. A área efetiva foi calculada através da seguinte expressão [6]:

$$
A_{\text {eff }}=\frac{\left(\iint\left|E^{2}\right| d x d y\right)^{2}}{\iint|E|^{4} d x d y}
$$

onde $E$ representa o campo elétrico.

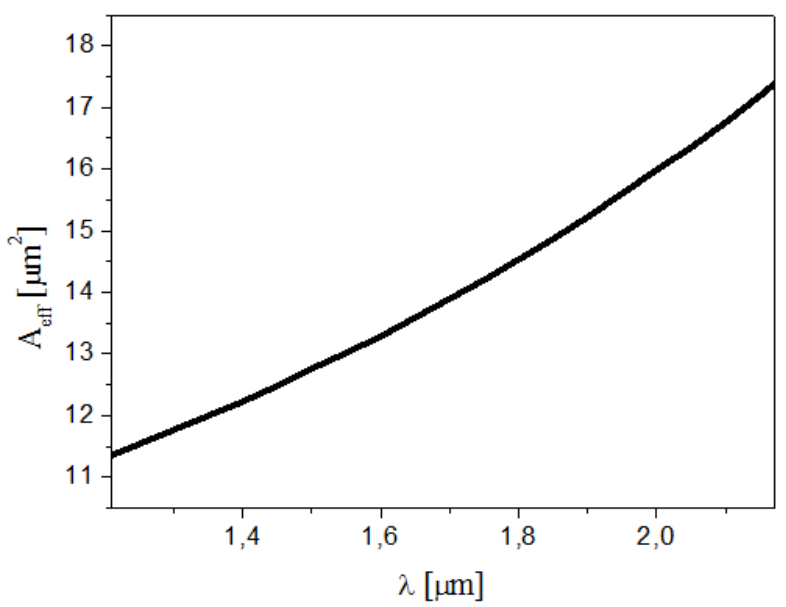

Fig. 5. Área efetiva do modo fundamental em função do comprimento de onda.

A fibra proposta neste trabalho apresenta características de uma fibra monomodal, como pode ser visto em Fig. 6. O modo fundamental corresponde ao modo $H_{11}^{x}$ e para os modos da Fig. 6 o índice de refração efetivo $\left(n_{\text {eff }}\right)$ é calculado através da relação $\beta / k_{0}$, obtido através da análise modal [8], e não são degenerados. Entretanto, resultados preliminares mostram que a presença do buraco de ar influencia na freqüência de corte dos modos propagantes em relação à fibra com núcleo sólido.

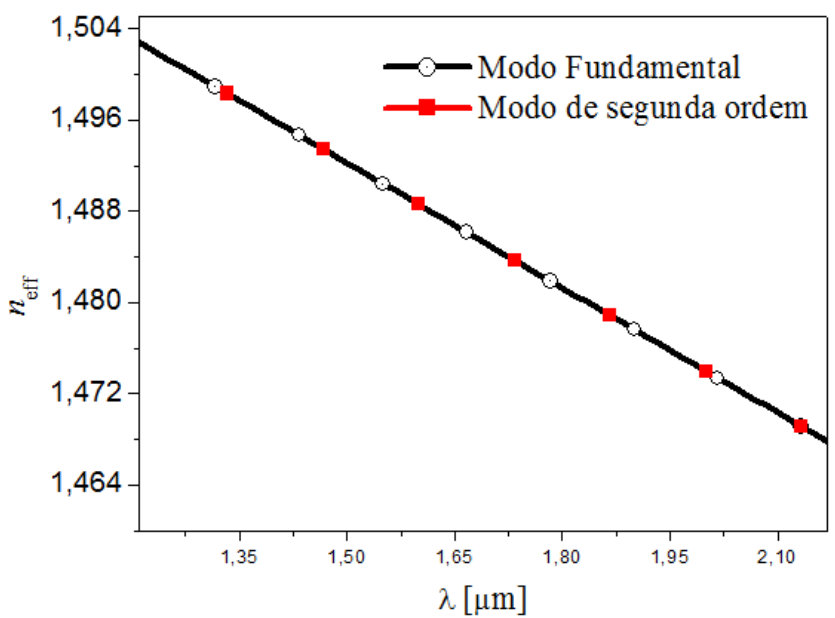

Fig. 6. Constante de propagação normalizada para os dois primeiros modos da fibra mostrada na Fig. 2.

A Fig. 7 mostra a distribuição de campo magnético para a componente $h_{x}$ do modo fundamental da estrutura mostrada na Fig. 2. 


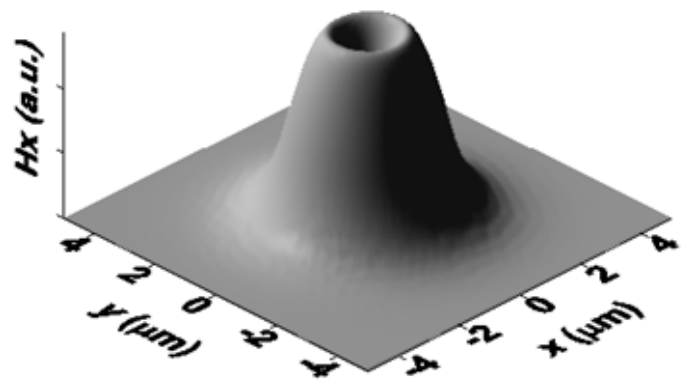

Fig. 7. Distribuição do campo magnético para a componente $h_{x}$.

\section{CONCLusõEs}

Neste trabalho, o método dos elementos finitos em conjunto com a técnica de otimização baseada em algoritmos genéticos, foram aplicados com sucesso na análise de um novo modelo de fibra óptica com dispersão cromática ultraplana, para uma extensa faixa de frequiência. Os resultados aqui obtidos mostram que, com uma estrutura mais simples, pode-se projetar um novo modelo de fibra óptica com dispersão cromática ultra-plana. A dispersão obtida neste trabalho variou entre $\pm 0,9 \mathrm{ps} /(\mathrm{nm} . \mathrm{km})$. O estudo feito poderá dar uma importante contribuição para aplicações em que o objetivo é obter dispositivos para compensação de dispersão cromática.

\section{AGRADECIMENTOS}

Os autores agradecem a UFERSA e UERN pelo apoio estrutural. Agradecemos também a FAPERN e CNPq pelo apoio financeiro.

\section{REFERÊNCIAS}

[1] A. Eason, B. Noble, e I. N. Sneddon, "On certain integrals of LipschitzHankel type involving products of Bessel functions", Phil. Trans. Roy. Soc. London, vol. A247, pp. 529-551, Abril 1955.

[2] V. Padgaonkar, A. Arbor, M. Lipson, e S. Pradhan, "Thermal effects in silicon based resonant cavit devices", http://www.nnin.org/doc/2004NNINreuVaidehee.pdf.

[3] R. Linden, "Algoritmos Genéticos", 2a Edição, Brasport, 428 p., ISBN: 9788574523736, 2008.

[4] J. P. da Silva, V. F. Rodríguez-Esquerre, H. E. Hernández-Figueroa, "Step index holey fiber for ultra-flattened chromatic dispersion", Proceedings of Integrated Photonics Research and Applications IPRA2006, Connecticut, 2006.

[5] D. Correia, V. F. Rodríguez-Esquerre, e H. E. Hernández-Figueroa, "Genetic-algorithm and finit-element approach to the synthesis of dispersion-flattened fiber", Microwave and Opt. Technol. Lett, 32, 245248, 2001.

[6] K. Saitoh e M. Koshiba, "Chromatic dispersion control in photonic crystal fiber: application to ultra-flattened dispersion", Opt. Express 11, 843-852, 2003.

[7] H. E. Hernández-Figueroa, F. A. Fernández, Y. Lu e J. B. Davies, "Vectorial finite element modelling of 2D leaky waveguides", IEEE Trans. Magn. 31, 1710-1712, 1995.

[8] D. Correia, J. P. da Silva e H. E. Hernández-Figueroa, “ Genetic algorithm and finit-element design of short single-section passive polarization converter", IEEE Photonics Technol. Lett, 15, 915-917, 2003.

[9] J. P. da Silva, H. E. Hernández-Figueroa e A. M. F. Frasson, "Vectorial finite-element BPM analysis for transverse anisotropic media", $\mathrm{J}$. Lightwave Technol., 21, 567-576, 2003.

[10] J. W. Fleming, "Material dispersion in lightguide glasses", Electron. Lett., 14 pp. 326-328, 1978. 